\title{
Una revisión propositiva a la política pública para el mejoramiento del gobierno en las IEs en Colombia
}

Recibido: febrero, 23 de 2018 - Aprobado: mayo, 14 de 2018

DoI: http://dx.doi.org/10.12804/revistas.urosario.edu.co/economia/a.7195

José Manuel Restrepo*
Henry Bradford $^{+}$
Alexander Guzmán ${ }^{\ddagger}$
María Andrea Trujillo§

Resumen

Las prácticas de gobierno en las instituciones de educación superior (IES) en Colombia han sido objeto de debate en la década actual. En este diálogo han participado el Ministerio de Educación Nacional, actores en los sistemas de gobierno de las IEs e investigadores que han desarrollado estudios sobre el tema en particular. En el marco de este debate, el Consejo Nacional de Educación Superior estableció la política pública para el mejoramiento del gobierno en las IES en noviembre de 2017. Esta política expresa aspectos relevantes para un buen gobierno en las IEs del país, pero deja de lado otros que consideramos fundamentales traer a discusión. Este artículo realiza un análisis crítico a la política pública promulgada en el año 2017 y sugiere al Ministerio de Educación Nacional nuevos aspectos

* Exrector, Universidad del Rosario y actual ministro de Comercio, Industria y Turismo.

+ Rector, Colegio de Estudios Superiores de Administración (CESA). Correo electrónico: henry.bradford@cesa.edu.co

$\ddagger$ Profesor titular, CESA. Correspondencia: Carrera 6 \# 34-51, CESA, Barrio La Merced, Bogotá (Colombia). Teléfono: (57+1) 3395300 ext. 1102. Correo electrónico: alexander.guzman@cesa.edu.co

§Profesora titular, cESA. Correo electrónico: maria.trujillo@cesa.edu.co

Cómo citar este artículo: Restrepo, J. M., Bradford, H., Guzmán, A., \&Trujillo, M. A. (2018). Una revisión propositiva a la política pública para el mejoramiento del gobierno en las IEs en Colombia. Revista de Economía del Rosario, 21(2), 219-246.

Dor: http://dx.doi.org/10.12804/revistas.urosario.edu.co/economia/a.7195 
que deben ser considerados en el establecimiento de lineamientos de gobierno para las IES colombianas.

Palabras clave: gobierno universitario, política pública, IES, buenas prácticas.

Clasificación JEL: I28, G30.

\title{
A Critical Review to the Public Policy for the Improvement of Governance Practices at HEIs in Colombia
}

\begin{abstract}
Good governance practices in Higher Education Institutions (HEIs) in Colombia have been a subject of discussion during the past decade. The Ministry of Education, key actors in HEIs' governance systems, and researchers with studies in this subject have participated in this dialogue. It was due to this debate that in November 2017 the National Council of Higher Education (CESU, by its acronym in Spanish) established the Public Policy for the improvement of Colombian HEIs' governance. This policy highlights relevant aspects for good governance at Colombian HEIs but leaves behind many others that we want to bring to discussion. This paper undertakes a critical review to the public policy enacted in 2017 and suggests to the Colombian Ministry of Education new issues to be considered in establishing governance guidelines for Colombian HEIs.
\end{abstract}

Keywords: University governance, public policy, HEIs, good practices.

JEL Classification: I28, G30.

\section{Uma revisão crítica da política pública para a melhoria das práticas de governança em IEs na Colômbia}

\section{Resumo}

As práticas de governança em instituições de ensino superior (IEs) na Colômbia foram objeto de debate na década atual. Este diálogo teve a participação do Ministério da Educação Nacional, atores dos sistemas de governo das IES e pesquisadores, que desenvolveram estudos sobre o tema em particular. Como resultado desse debate, o Conselho Nacional de Educação Superior estabeleceu a política pública para o melhoramento de governança nas IES em novembro de 2017. Esta política expressa aspectos relevantes para uma boa governança nas IEs do país, mas deixa de lado outros que consideramos fundamentais para debate. Este artigo realiza uma análise crítica da política pública promulgada no ano de 2017 e sugere ao Ministério da Educação Nacional novos aspectos que devem ser considerados no estabelecimento de diretrizes de governança para as IEs colombianas.

Palavras-chave: governança universitária, política pública, IEs, boas práticas.

Classificação JEL: I28, G30. 


\section{Introducción}

En concordancia con la importancia que revisten las buenas prácticas de gobierno en las instituciones de educación superior (IES), en Colombia la reflexión alrededor del tema ha surgido de manera paulatina y se ha intensificado en los últimos cinco años. Uno de los primeros trabajos en abordar el tema es el de Cárdenas y Gutiérrez (2004), quienes resaltan limitaciones en aspectos jurídicos asociados a la actualización y contenido de los estatutos de las IES privadas y, específicamente, la carencia de un régimen de inhabilidades para los miembros de sus consejos directivos o superiores. De igual forma, señalan problemas en relación con el manejo financiero y la contratación, el pago y liquidación de los docentes, y limitaciones en algunas de ellas referentes a la acreditación de calidad y rendición de cuentas.

Posteriormente, el trabajo de Restrepo, Trujillo y Guzmán (2012), resultado de una investigación financiada por el Ministerio de Educación Nacional en el marco de la Convocatoria para Realización de Estudios sobre Educación Superior del año 2012, vitaliza la discusión sobre la necesidad de buenas prácticas de gobierno en las IES colombianas. Este estudio llevó a cabo entrevistas en profundidad a rectores de diferentes IEs en el país.

Las conclusiones de Restrepo y colaboradores señalan la necesidad de definir perfiles con unas condiciones mínimas de formación y conocimiento del sector para los cargos de alta gerencia y los consejeros (miembros de juntas directivas), establecer claramente las responsabilidades legales y morales que asumen estos, ofrecer capacitación especializada en temas de gobierno para los consejeros, garantizar independencia en los consejos y propender por la implementación de prácticas que permitan reuniones efectivas y faciliten el aporte asertivo de los miembros del consejo.

Es necesario resaltar que las recomendaciones en este estudio no se limitan a sugerir mejores prácticas en los consejos. Los autores destacan aspectos como la concentración del poder en algunas iEs y los riesgos de extracción de rentas a través de prácticas cuestionables y que no reconocen la naturaleza de estas instituciones como organizaciones sin ánimo de lucro; discuten los riesgos del exceso de democracia y de una democracia no basada en méritos en las universidades públicas; argumentan la necesidad por parte de las IES de contar con códigos de buen gobierno y políticas relacionadas con la gestión de los conflictos de intereses; y concluyen con una reflexión sobre la pertinencia del establecimiento de unos mínimos de gobierno corporativo para las IEs en Colombia, y la necesidad de incrementar las exigencias en términos de transparencia y revelación de la información, incluida la financiera. 
A partir de entonces, se evidencia una dinámica de trabajo relevante para el sistema de educación superior colombiano en este particular. Por ejemplo, en el año 2013 el Ministerio de Educación Nacional (MEN) publicó la Guía para la creación e implementación de códigos de buen gobierno en las instituciones de educación superior (MEN, 2013). A través del documento, el Ministerio resalta la relevancia del gobierno corporativo para la gestión estratégica de las universidades, la gestión de riesgos y el ambiente de control. Además, hace explícito lo que el MEN considera un código de buen gobierno universitario y despliega el contenido que deberían tener las instituciones que deciden promulgar su código. Los aspectos por contemplar en esta propuesta son diversos, y aquí solo se mencionan algunos de ellos, como la orientación estratégica, las políticas de buen gobierno deseables, las políticas de relación con los órganos de control internos y externos, las políticas de administración y gestión (por ejemplo, de conflictos de interés, y algunas específicas de grupos de interés estratégicos, como los empleados, la comunidad y el medio ambiente), y normas explícitas para la administración, seguimiento y evaluación del código.

En el año 2014 se presentan dos hechos relevantes en la evolución de la discusión del gobierno universitario en Colombia. Primero, el MEN y la Escuela de Gobierno y Ética Pública de la Pontificia Universidad Javeriana publican el informe técnico titulado "Recomendaciones para una política pública sobre buen gobierno universitario en Colombia" (MEN y PUJ, 2014). Este esfuerzo, liderado por el abogado y secretario general de la Universidad Javeriana, Jairo Cifuentes Madrid, presenta las conclusiones derivadas de cinco mesas de trabajo a las que se convocaron directivos de IEs colombianas, públicas y privadas, para identificar aspectos fundamentales para la construcción de una política pública de buen gobierno universitario para el país.

Las conclusiones de las mesas de trabajo resaltan la importancia de aspectos del gobierno universitario como la dirección estratégica de las instituciones, las relaciones con grupos de interés internos y externos, la rendición de cuentas y transparencia, y las políticas para la administración de conflictos de interés. Si bien las recomendaciones del documento no son tan detalladas como sucede en los códigos de buen gobierno alrededor del mundo, destacan aspectos fundamentales en el gobierno universitario y una preocupación generalizada en aquellos que participan del gobierno de las IEs en el país.

El segundo hecho relevante del año 2014 fue la promulgación de la Ley 1740 del 23 de diciembre de ese año, por la cual se establecen las normas de inspección y vigilancia de la educación superior en Colombia. Esta ley cobija a todas las IEs del país y pretende velar por la calidad y continuidad del servicio público de educación superior. La ley en mención faculta al MEN 
para realizar inspección mediante la solicitud y evaluación de todo tipo de información de la institución, especialmente actos y contratos, la verificación de la información entregada a la comunidad estudiantil activa y potencial (por ejemplo, en los procesos publicitarios), y la información inherente a los reportes y estados financieros. La vigilancia faculta al MEN a efectuar ejercicios de auditoría mediante visitas a la IES, especialmente sobre procedimientos financieros y contables, pero no limitados a este aspecto en particular, verificar el cumplimiento de la ley en la prestación del servicio, solicitar informes detallados y realizar control a las actuaciones de los representantes legales, equipo de alta gerencia y miembros de órganos de dirección.

De acuerdo con la Ley 1740, las acciones que puede tomar el MEN van desde solicitar el desarrollo e implementación de planes de mejoramiento hasta tomar el control administrativo de la IEs enviando delegados a los órganos de gobierno. Las sanciones además pueden ser para los directivos, representantes legales, equipo de alta gerencia o revisores fiscales, y contempla sanciones monetarias y de separación de las funciones, así como imposición de inhabilidades hasta por diez años. Las IEs se exponen a multas, suspensión o cancelación de programas académicos o registros calificados, y suspensión o cancelación de la personería jurídica.

En años más recientes el interés sobre el tema en particular incluso ha suscitado la realización y culminación de tesis de maestría (Prada-Rangel, 2014; Méndez-Beltrán, 2015) o de doctorado (Flórez-Parra, 2015), que han conducido a publicaciones en revistas académicas (Flórez-Parra et al., 2017, 2018). De igual manera, Restrepo y colaboradores han continuado con la agenda de investigación al respecto. Por ejemplo, en Bradford et al. (2018a) se hace un análisis al sistema de gobierno a nivel organizacional en las IEs colombianas. Los autores argumentan que, dado la inexistencia de propietarios en organizaciones sin ánimo de lucro, se espera que los consejos superiores o directivos cuenten con todo el poder y representen el máximo órgano de gobierno. Bajo esta situación y sin mecanismos de control efectivos, los consejos pueden hacer un mal uso de los recursos y distraer a la institución del cumplimiento de sus objetivos fundacionales. Sin embargo, los autores encuentran que en el $76 \%$ de las IEs privadas en Colombia existe una asamblea (de fundadores, miembros o representantes) como el máximo órgano de gobierno, con la potestad de nombrar el consejo. Bradford et al. (2018a) discuten la razón de ser, estructura y funciones de estos órganos, su poder excesivo y la necesidad de establecer un balance de poderes apropiado para evitar extracción de rentas y comportamientos autointeresados de parte de los miembros de estos órganos de gobierno en las IEs privadas colombianas. 
Otro artículo de los mismos autores resalta lo común que resulta el involucramiento familiar en las IEs privadas del país, las cuales, al ser organizaciones sin ánimo de lucro, en la gran mayoría de casos cuentan con familias que deciden financiar las instituciones en su etapa de formación, como una manera de contribuir al país y ejercer actividades de responsabilidad social. Sin embargo, en ocasiones este involucramiento familiar puede derivar en situaciones poco deseables, como la extracción de rentas por parte de la familia a través de transacciones con partes relacionadas y realizadas a precios que no corresponden a los del mercado. Por lo tanto, Bradford et al. (2018b) sugieren la necesidad de establecer unos mínimos de gobierno corporativo para regular el involucramiento familiar de los fundadores en las IEs, potenciando las externalidades positivas que esta situación genera y mitigando los posibles problemas de agencia y conflictos de interés asociados a ella. Para soportar sus argumentos, los coautores hacen una breve reseña del caso de la Fundación San Martín.

El último hecho de vital importancia, y que motiva la escritura de este artículo, está en que el Consejo Nacional de Educación Superior (CESU), mediante el Acuerdo 2 del 8 de noviembre de 2017, estableció la política pública para el mejoramiento del gobierno en las IEs colombianas. Esta política expresa de manera concreta aspectos que el cESU considera relevantes para un buen gobierno en las IEs del país, pero deja aspectos de lado que consideramos fundamentales traer a discusión. Por lo tanto, en la siguiente sección resaltaremos las fortalezas de esta política, para luego emitir unas recomendaciones que permitirían hacer más comprensiva la propuesta del CESU para las IES del país. Finalmente, presentamos un comentario de cierre.

\section{Fortalezas de la política pública para el mejoramiento del gobierno en las IES colombianas}

\subsection{Generalidades}

El CESU representa un organismo de gobierno nacional vinculado al MEN, con funciones de planificación, coordinación, recomendación y asesoría, por lo que está facultado para proponer políticas para la educación superior en el país. Por medio del Acuerdo 2 de 2017 (de ahora en adelante el Acuerdo), y en concordancia con la Ley 30 de 1992, el CESU reconoce como parte de la autonomía universitaria el derecho de las universidades a darse y modificar sus estatutos, designar sus autoridades académicas y administrativas, adoptar sus regímenes correspondientes y establecer, arbitrar y aplicar sus 
recursos para el cumplimiento de su misión social y función institucional. Sin atentar contra dicha autonomía, el cEsu establece la política pública para el mejoramiento del gobierno en las IEs, buscando cualificar el gobierno de estas y asegurar calidad y pertinencia en el cumplimiento de sus funciones. Específicamente, este Acuerdo define principios de buen gobierno, presenta recomendaciones o lineamientos sobre buenas prácticas en el contexto del gobierno universitario, y hace explícitas herramientas para fortalecer los máximos órganos colegiados de gobierno en las IEs.

El Acuerdo presenta inicialmente conceptos sobre buen gobierno, definiendo autonomía universitaria, gobierno institucional, código de buen gobierno, máximo órgano colegiado de gobierno, gobernabilidad, gobernanza, grupos de interés y rendición de cuentas. Queremos resaltar especialmente la definición de gobierno institucional como las políticas, estrategias, decisiones, estructuras y procesos, encaminadas al cumplimento de la misión con criterios de ética, eficiencia, eficacia, calidad, integridad y transparencia. Además, el Acuerdo otorga un papel relevante a la rendición de cuentas, a través de la cual los rectores, representantes legales y los órganos de gobierno responden e informan de manera periódica y planeada por el manejo eficiente y eficaz de los recursos, y, en general, por los resultados de la institución.

La definición puntual sobre gobierno a nivel institucional resulta un buen complemento a definiciones previas sobre gobierno en la educación superior (OCDE, 2008), y ofrece claridad respecto a que el sistema de gobierno no está representado únicamente por normas, estructuras organizacionales o procesos, sino que es una combinación de todo lo anterior con una orientación de productividad, integridad y transparencia, lo que permite el establecimiento de buenas prácticas de gobierno.

Por otra parte, la revelación de información y transparencia ha sido ampliamente discutida como una práctica que permite reducir las asimetrías de información y exponer a las organizaciones a un mayor nivel de escrutinio por parte de sus grupos de interés. Las asimetrías de información emergen naturalmente porque los miembros de los órganos de gobierno y del equipo de alta gerencia tienen más información respecto al quehacer de las organizaciones en comparación con los demás grupos de interés. Cuando las organizaciones son opacas afectan el funcionamiento de los mercados (Akerlof, 1970) y se hace más complejo el obtener recursos para desarrollar el objeto social de estas. En este contexto, la construcción de reputación y confianza es importante para las instituciones bien gobernadas (Gomes, 2000), y lo anterior es posible a través de mecanismos de revelación que permitan señalar las buenas prácticas a los diferentes grupos interesados (Spence, 1973, 1974). Por lo tanto, un acierto del Acuerdo está en el énfasis que da a esta práctica en particular. 
Revelación de información y transparencia hacen referencia a las buenas prácticas adoptadas para reducir la opacidad de las organizaciones e incentivar una comunicación asertiva con los diferentes grupos de interés. Tal como lo proponen Trujillo y Guzmán (2015), si el lector se pregunta cuál es la diferencia entre revelación y transparencia, la respuesta es sencilla. La primera responde a los requerimientos de ley; y la segunda, a la adopción voluntaria de buenas prácticas para mitigar las asimetrías de información entre el equipo de alta gerencia y los distintos grupos de interés de las organizaciones, lo que incrementa la confianza por parte de los externos y conduce a mayor reputación para la organización. Sin embargo, es común que estos términos sean usados indistintamente.

\subsection{Principios de buen gobierno}

Otro punto a favor del Acuerdo está en resaltar unos principios orientadores de buen gobierno en el que se incluye la prevalencia de los intereses institucionales, la toma de decisiones con base en evidencias, la declaración de los grupos de interés y la fluida relación con ellos, la gestión eficiente y eficaz de recursos, y la cultura de rendición de cuentas. La prevalencia de los intereses institucionales hace referencia a la necesidad de salvaguardar la institución en contra de intereses o motivaciones particulares e invita a hacer explícitos y a administrar eficientemente los conflictos de interés. Los problemas de agencia bajo los cuales priman intereses particulares que pueden afectar las organizaciones en general han sido estudiados por Jensen y Meckling (1976), Myers (1977), Fama y Jensen (1983), Shleifer y Vishny (1986) y Villalonga et al. (2015) principalmente, y en todos estos artículos se hace evidente cómo los intereses particulares de los administradores, de los miembros de los órganos de gobierno y de quienes ejercen la propiedad, pueden llevar a extracción de rentas y a atentar contra la viabilidad de las organizaciones. Es por ello que un manejo adecuado de los conflictos de interés constituye una base fundamental para el buen gobierno.

El segundo principio, orientado a la toma de decisiones con base en evidencias, recomienda a los máximos órganos de gobierno contar con un direccionamiento estratégico y con herramientas de gestión, como tableros de mando, una batería de indicadores e información proveniente de sistemas internos y externos. La formulación estratégica con participación de los consejos superiores o directivos resulta fundamental para las IES. Según Andrews (1971), la estrategia corporativa representa una función vital de la junta directiva. Para el autor, la participación efectiva de la junta en el proceso de planeación estratégica es fundamental para el desempeño financiero y supervivencia de 
las organizaciones. Investigaciones empíricas, como la de Siciliano (2005), demuestran que en la práctica estos órganos de gobierno participan en la definición de la estrategia y el seguimiento a su implementación. Además, una comunicación efectiva entre los consejos superiores o directivos y los rectores incrementa la probabilidad de que el control que ejercen los primeros no se limite al desempeño financiero, y que, por el contrario, el acompañamiento de los consejos se enfoque en la formulación y despliegue de una estrategia coherente para la IEs (Hendry y Kiel, 2004).

El tercer principio orientador de buen gobierno señala la necesidad para los máximos órganos de gobierno de hacer explícitos los grupos de interés de la IEs, en una visión amplia que incluya grupos internos y externos, y la importancia de identificar los intereses, requerimientos y expectativas de estos, para que la estrategia de relacionamiento con cada uno de ellos se desarrolle de manera coherente y comprensiva. Desde este enfoque, el gobierno de las organizaciones debe tener en cuenta no solo las expectativas de los grupos de interés con mayor poder de influencia o control. Este principio de gobierno está en concordancia con las discusiones que se vienen desarrollando en el sector corporativo. Tirole (2001) sostiene que la orientación tradicional basada en la generación de valor para los propietarios es demasiado reducida para realizar un análisis desde el punto de vista económico del gobierno corporativo, y define el gobierno corporativo como "el diseño de instituciones que inducen $\mathrm{u}$ obligan a la alta gerencia a internalizar el bienestar de los grupos de interés" (2001, p. 4).

El cuarto principio, que hace referencia a la gestión eficiente y eficaz de recursos, reconoce la importancia de hacer un buen uso de los recursos que las IES reciben del Estado o de la sociedad en general. Tal como lo discutiremos en las oportunidades para hacer más comprensiva esta política pública, bajo los sistemas de gobierno corporativo es fundamental garantizar un ambiente de control adecuado, buscando que los recursos financieros sean administrados correctamente. Los códigos de buen gobierno a nivel país y a nivel organizacional regularmente incluyen recomendaciones sobre las funciones de control interno y auditoría, así como la prevención del fraude (Trujillo y Guzmán, 2017). Aun cuando la política aquí analizada incluye como principio de buen gobierno la gestión eficiente y eficaz de los recursos, no desarrolla de manera explícita recomendaciones y no brinda herramientas al respecto.

Como lo mencionamos anteriormente, el Acuerdo resalta la relevancia de la rendición de cuentas. Esto no se limita a las definiciones proporcionadas en el glosario, sino que es subrayado en el quinto principio de buen gobierno, bajo el cual se hace explícita la importancia de establecer una cultura 
orientada ${ }^{1}$ a informar a los grupos de interés sobre la gestión de los recursos, el cumplimiento de los objetivos trazados, el desarrollo de los proyectos, el manejo de recursos y, en especial, de las expectativas cumplidas y no cumplidas. Lo más desafiante ante este principio es la identificación concreta de los grupos de interés, ya que en un sentido amplio son los individuos o colectivos los que pueden afectar o verse afectados por el desarrollo de los objetivos de la organización (Freeman, 1984). Sin embargo, que resulte una tarea compleja no implica que no sea necesaria. El reconocimiento de la existencia y relevancia de los diferentes grupos de interés permite a los consejeros y rectores establecer un diálogo productivo y políticas claras para generar valor compartido. La tabla 1 presenta una síntesis de estos principios de buen gobierno, consignados en el Acuerdo 2 de 2017.

Tabla 1. Principios de buen gobierno en el Acuerdo 2 de 2017

\begin{tabular}{|c|c|c|}
\hline & Principio & Conceptualización \\
\hline 1. & $\begin{array}{l}\text { Prevalencia de los Inte- } \\
\text { reses Institucionales }\end{array}$ & $\begin{array}{l}\text { Los miembros del Máximo Órgano de Gobierno institucional de- } \\
\text { ben salvaguardar los objetivos de la educación superior según la } \\
\text { Ley, su Misión y el Proyecto Educativo Institucional. }\end{array}$ \\
\hline 2. & $\begin{array}{l}\text { Toma de decisiones } \\
\text { con base en evidencias }\end{array}$ & $\begin{array}{l}\text { El proceso de toma de decisiones debe enmarcarse y ser consecuente } \\
\text { con la realidad de la institución y el proyecto educativo. El mismo } \\
\text { debe sustentarse en herramientas de gestión para su seguimiento. }\end{array}$ \\
\hline 3. & $\begin{array}{l}\text { Declaración de los gru- } \\
\text { pos de interés y fluida } \\
\text { relación con ellos }\end{array}$ & $\begin{array}{l}\text { Los máximos órganos de gobierno deben identificar los grupos de } \\
\text { interés de la institución y las estrategias para el relacionamiento } \\
\text { con los mismos. }\end{array}$ \\
\hline 4. & $\begin{array}{l}\text { Gestión eficiente y efi- } \\
\text { caz de los recursos }\end{array}$ & $\begin{array}{l}\text { Las instituciones de educación superior deben dar cuenta de una } \\
\text { gestión eficiente y transparente de los recursos que le son entre- } \\
\text { gados por parte del Estado o la sociedad. De la misma manera, } \\
\text { deberán acudir a diversas fuentes de financiación y mitigar la } \\
\text { dependencia de una única fuente. }\end{array}$ \\
\hline 5. & $\begin{array}{l}\text { Cultura de rendición } \\
\text { de cuentas }\end{array}$ & $\begin{array}{l}\text { Las instituciones de educación superior tienen el deber ético de } \\
\text { construir una cultura de rendición de cuentas orientada a estable- } \\
\text { cer un diálogo continuo con los grupos de interés en relación con } \\
\text { la gestión realizada en el ejercicio de su misión. }\end{array}$ \\
\hline
\end{tabular}

Fuente: Acuerdo 2 de 2017, por medio del cual se establece la política pública para el mejoramiento del gobierno en las instituciones de educación superior en Colombia.

1 De acuerdo con Corbally y Sergiovanni (1984), una definición estándar de cultura podría incluir un sistema de valores, símbolos y significados compartidos por los integrantes de una organización, incluyendo la personificación de esos valores, símbolos y significados en objetos materiales y prácticas que se convierten en tradición. Por lo tanto, una cultura incluye costumbres y tradiciones, relatos históricos (míticos o reales), entendimientos tácitos, hábitos, normas y expectativas, significados comunes asociados con objetos fijos y rutinas establecidas, así como suposiciones compartidas, entre otros. 


\subsection{Recomendaciones y herramientas}

El Acuerdo aquí analizado incluye recomendaciones específicas para un buen gobierno. Las primeras recomendaciones se ofrecen sobre las políticas institucionales, bajo las cuales se hace énfasis en la necesidad de establecer sistemas de información para el seguimiento al desarrollo de la razón de ser de las IES, así como en la necesidad de evaluar la pertinencia y contribución de los mecanismos de elección directa, puesto que la utilización de estos no constituye en sí misma un buen gobierno. Además, se invita a asegurar que la toma de decisiones sea llevada a cabo por las personas con mayor conocimiento sobre ellas. Estas recomendaciones son fundamentales para evitar los riesgos asociados a los excesos de democracia y, a su vez, garantizar una democracia calificada (Restrepo et al., 2012).

Respecto a los máximos órganos de gobierno, las recomendaciones señalan la importancia de su presidente, como vocero del colectivo. Adicionalmente, resaltan dentro de sus funciones la selección, nombramiento, apoyo, evaluación y solicitud de retiro de la máxima autoridad personal de gobierno, que generalmente es el rector. Conforme con Fama y Jensen (1983), son funciones inherentes a las juntas directivas la selección, remuneración y despido de la alta gerencia. Un buen gobierno debe otorgar a los consejos la potestad de sancionar al rector cuando este actúa con negligencia, garantizando un equilibrio de poderes adecuado para la IEs en procura del bienestar colectivo.

Otra recomendación fundamental relacionada con los máximos órganos de gobierno hace mención a la participación por mecanismos de elección directa, los cuales, según el Acuerdo, deben estar orientados por criterios de meritocracia. Además, si la participación es por designación, debe realizarse siguiendo la misma lógica. Lo anterior pretende nuevamente evitar los problemas asociados a los excesos de democracia. Para Huntington (1975), una institución donde el nombramiento de los profesores está sujeto a la aprobación de los estudiantes puede ser una institución más democrática, pero no necesariamente una mejor institución. Así mismo, se busca establecer en las IES una democracia calificada, construida sobre la meritocracia, y se propende por mejores perfiles en los consejos superiores o directivos (Restrepo et al., 2012).

Para estos órganos colectivos, la política pública sugiere igualmente algunas recomendaciones orientadas a su buen funcionamiento, como procurar sesiones efectivas con agendas estratégicas, procedimientos definidos y mecanismos que faciliten la participación de los consejeros. Posteriormente, las recomendaciones se centran en los consejeros. Para ellos aclara su papel de fideicomisarios, procurando la continuidad de la IES, la calidad en el servicio prestado, la no intromisión en las tareas administrativas, la defensa 
del interés común antes que el de algún grupo o individuo en particular, la asistencia periódica a las reuniones y la capacitación permanente. Además, invita a no establecer relaciones comerciales con la institución y a revelar cualquier conflicto de interés. Todo lo anterior en concordancia con lo que se espera de los integrantes de los máximos órganos de gobierno (Fama y Jensen, 1983; Restrepo et al., 2012; Trujillo et al., 2015).

Las recomendaciones igualmente sugieren que los procesos para la elección de la máxima autoridad personal de gobierno consideren mecanismos de participación de la comunidad, sin ser vinculantes ni exclusivos como mecanismos de elección, y que la IEs considere no solo a egresados o funcionarios de la institución. Lo anterior pretende evitar el nepotismo y el favorecimiento a algunos individuos por sus relaciones interpersonales. Se busca facilitar el acceso a un grupo más amplio de capital humano al momento de seleccionar los rectores, incrementando la probabilidad de contar con alguien idóneo para este cargo (Coffee, 1999; Bertrand y Schoar, 2006).

La política culmina ofreciendo una discusión de lo que el cESU considera herramientas para el buen gobierno, como la actualización normativa, el seguimiento a procesos de calidad académica, la batería de indicadores, el manual de rendición de cuentas de los máximos órganos de gobierno y el manual de inducción para sus miembros. De estas herramientas queremos destacar aquellas claramente relacionadas con prácticas de buen gobierno, como la actualización y diseño de una normativa interna adecuada, con políticas explícitas para hacer más eficiente el sistema de gobierno interno de la IES, así como el diseño de una política de inducción para los miembros de los consejos, para garantizar su rápida adaptación y potenciar su capacidad de contribución enmarcada en la realidad de la institución que acompañan (Restrepo et al., 2012; Guzmán y Trujillo, 2016).

Luego de reconocer en esta sección las fortalezas de la política pública para el mejoramiento del gobierno de las IES acordada por el CESU, en el siguiente aparte de este artículo haremos explícitos los aspectos desatendidos que hemos identificado en el análisis a este Acuerdo y que consideramos de vital importancia para el buen gobierno en las IES colombianas.

\section{Oportunidades para hacer más comprensiva la política pública para el mejoramiento del gobierno en las ies colombianas}

\subsection{Sobre las políticas institucionales ausentes}

Los lineamientos en el ámbito internacional sobre buenas prácticas resaltan generalmente políticas indispensables para un buen gobierno. De ellas podemos 
mencionar tres en particular: la política para el manejo de los conflictos de intereses, la política de transacciones con partes relacionadas y la política de relevación de información, todas ellas de potestad de las juntas directivas, lo que, para el caso particular de las IES, representan políticas que deben ser definidas por los consejos superiores o directivos.

Los conflictos de interés en el ámbito organizacional han sido estudiados por teóricos relevantes como Jensen y Meckling (1976). Para estos autores, las organizaciones representan un escenario en el que los intereses en conflicto de diferentes individuos (algunos representando otras organizaciones) llegan a un equilibrio en un marco de relaciones contractuales a través de un proceso complejo. Además, afirman que siempre que se administran recursos de terceros es de esperarse algo de negligencia y un comportamiento orientado al interés particular y no al bienestar común. Denis (2001), a través de una reseña a 25 años de investigación en gobierno corporativo, muestra cómo los mecanismos de gobierno están diseñados para mitigar los conflictos de interés. De ahí la importancia de hacer explícita una normativa interna que haga referencia a estos conflictos y que fije los lineamientos para gestionarlos.

De acuerdo con Guzmán y Trujillo (2016), la política para la gestión de los potenciales conflictos de interés debe hacer explícita su definición, tratamiento, inhabilidades e incompatibilidades, políticas de divulgación y mecanismos de resolución de las posibles controversias que se puedan generar en el interior de la organización. También se puede incluir un apartado que haga mención a las normas internas de la compañía relacionadas con la mitigación de estos conflictos.

Uno de los referentes más relevantes en el país y que ofrece recomendaciones para buen gobierno es el Código de Mejores Prácticas Corporativas, promulgado por primera vez por la Superintendencia Financiera en el año 2007 y actualizado en el año 2014. Este código ha servido de referente en el ámbito nacional para diferentes sectores, como el sector salud y el sector solidario. En las recomendaciones de mejores prácticas consagradas en la Circular Externa 28 de 2014, se sugiere a las juntas directivas establecer una política $\mathrm{y}$ un procedimiento definido y formalizado en la normativa interna para el conocimiento, administración y resolución de las situaciones de conflicto de interés, ya sean directos o indirectos a través de partes vinculadas, que puedan afectar a los miembros de la junta directiva y demás administradores. Igualmente, sugiere a los integrantes de los órganos de gobierno y a la alta dirección informar periódicamente la existencia de conflictos de interés que los afecten. Lo anterior señala la necesidad de supervisar y administrar este tipo de conflictos, especialmente cuando afectan a personas que ostentan poder y participan activamente en la toma de decisiones de las organizaciones. 
Respecto a las transacciones con partes relacionadas, Gordon et al. (2004) las definen como operaciones que se llevan a cabo entre la organización y alguno de los miembros del equipo directivo, de los órganos de gobierno o de los accionistas principales. Los autores encuentran que están transacciones pueden ir desde préstamos otorgados por la organización, hasta la compra de bienes o servicios provistos por altos ejecutivos o miembros de los órganos de gobierno. Además, los autores demuestran el efecto negativo de estas operaciones sobre el desempeño financiero de las organizaciones. Nekhili y Cherif (2011) igualmente concluyen que este tipo de transacciones afecta de manera negativa el desempeño financiero de las organizaciones, y también ofrecen evidencia de que sugiere que buenas prácticas de gobierno corporativo y un ambiente de control fuerte, con un auditor externo reconocido, disminuyen la probabilidad de ocurrencia de este tipo de transacciones.

La política de transacciones con partes relacionadas o de operaciones con partes vinculadas es fundamental y complementa la política para la administración de conflictos de interés. Nuevamente, la Circular Externa 28 de 2014 recomienda la definición de esta política por parte de las juntas directivas. Una adaptación de la definición de una parte vinculada o relacionada, tomada de la Guía de gobierno corporativo para sociedades cerradas y de familia (ssoc, 2009), es la siguiente: por parte vinculada o relacionada se entiende las personas jurídicas o naturales relacionadas con los fundadores, integrantes de los órganos de gobierno, rector, vicerrectores, decanos y otros ejecutivos clave de la institución. Ejemplos de estas, respecto de las personas naturales, pueden ser: a) El cónyuge o compañero permanente o personas con análoga relación de afectividad, como los novios. b) Los padres, suegros, hijos, hijastros, hermanos, hermanastros y cuñados. c) Los padrastros, madrastras, yernos y nueras. d) Los socios en negocios o emprendimientos, o aliados comerciales del rector, vicerrectores, decanos y otros ejecutivos clave, en actividades con ánimo de lucro. Ejemplos de estas, respecto de las personas jurídicas pueden ser: a) Las sociedades que tengan la consideración de algún grado de asociación, como empresa del grupo, asociada a un mismo conglomerado empresarial. b) Las sociedades que compartan algún miembro de junta directiva o las IEs que compartan algún miembro de consejo superior o directivo. c) Las sociedades que tengan como accionistas o socios a los fundadores, integrantes de los órganos de gobierno, rector, vicerrectores, decanos y otros ejecutivos clave de la institución.

Una política formal de transacciones con partes relacionadas define cuándo una operación es considerada como tal y qué acciones deben realizarse para asegurar que no sean perjudiciales, sean necesarias para la institución y se realicen a precio y condiciones de mercado. Sostener políticas adecuadas 
en esta materia permite que las instituciones se muestren transparentes ante la mirada externa, es decir, ante los grupos de interés y entes reguladores. Conforme con la Circular Externa 28 de 2014, una política al respecto debe abordar por lo menos tres aspectos. El primero es la valoración de la operación, para garantizar la igualdad de trato de los diferentes grupos de interés y el respeto a las condiciones de mercado. El segundo es la aprobación, que debe estar a cargo de los consejos superiores o directivos sin participación de la parte interesada, y si es el caso, dependiendo de la cuantía y de la estructura de gobierno de la IEs, del máximo órgano colectivo, en caso de existir otro diferente a los consejos y con mayor autoridad. El tercer aspecto es la revelación, que implica la divulgación de este tipo de operaciones a los grupos de interés. De acuerdo con la Circular Externa 28 de 2014, este tipo de operaciones no son negativas necesariamente, y en ocasiones pueden ser económicamente beneficiosas y generadoras de valor, pero, en otras oportunidades, pueden generar un riesgo de abuso o de apropiación indebida. Aquí radica la importancia de la política y la transparencia alrededor de estas operaciones.

Finalmente, respecto a la revelación de información, además de los argumentos y trabajos teóricos citados previamente, Católico (2012) argumenta que esta práctica de gobierno constituye la base para la rendición de cuentas y encuentra también que factores como la calidad en la gestión de las IES, la financiación por parte del Estado y la calidad en los resultados de investigación están relacionados con buenas prácticas de revelación por parte de las IES colombianas. Por otra parte, Flórez-Parra et al. (2017) resaltan el incremento en las demandas de la sociedad en general en los últimos años por un mayor nivel de transparencia y de rendición de cuentas por parte de las IEs. Para estos autores, las universidades colombianas han venido implementando mecanismos que responden a estas presiones del entorno y encuentran una relación entre las prácticas de revelación y el buen gobierno en estas instituciones.

La política de revelación de información a nivel institucional debe ser aprobada por el consejo superior o directivo, y debe identificar el departamento o unidad responsable en el interior de la IEs de proponer y desplegar la política, la información por revelar, la forma de hacerlo, los grupos de interés a quienes se debe revelar información, los mecanismos que garantizan la calidad y pertinencia de la información revelada, y los procedimientos para determinar qué información es de carácter confidencial para la institución (Restrepo et al., 2012; Circular Externa 28 de 2014).

Todas estas políticas pueden recogerse en el Código de Buen Gobierno de la IEs, el cual constituye un documento único que busca formalizar, sintetizar y dar a conocer las normas relacionadas con el buen gobierno establecidas por una organización. La consolidación de las disposiciones voluntarias y 
regulatorias adoptadas por las IEs evidencia el compromiso adquirido por los órganos de gobierno en busca de una gestión que atienda los diferentes grupos de interés (Guzmán y Trujillo, 2016).

\subsection{Sobre las recomendaciones asociadas a los máximos órganos de gobierno}

Aun cuando la política pública aquí analizada orienta la mayoría de recomendaciones a los consejos superiores o directivos, es deseable incluir dos aspectos adicionales. Primero, en lugar de sugerir como recomendación la convocatoria periódica de las reuniones de estos órganos de gobierno, con sesiones efectivas y agendas estratégicas, es más comprensivo sugerir a las IES el establecimiento de reglamentos con normas detalladas para el funcionamiento de los órganos de gobierno. Huse (2000) resalta la importancia de la formalización de las reglas de los órganos de gobierno, no solo por adopción voluntaria, sino como respuesta a los cambios regulatorios que buscan mayor formalidad en las prácticas de gobierno organizacionales, y, especialmente, en la claridad sobre las obligaciones de los equipos de alta gerencia versus las obligaciones de los órganos que los asesoran y supervisan.

Según Guzmán y Trujillo (2016), los reglamentos permiten garantizar el adecuado funcionamiento de los órganos de gobierno, mantener una delineación clara de sus funciones, establecer las normas relacionadas con su composición y con el desarrollo de reuniones efectivas.

Se recomienda incorporar en el reglamento el objeto y ámbito de aplicación, la descripción de los tipos de reuniones, el lugar en que se deben desarrollar, el número de reuniones o cronograma anual, los medios para realizar la convocatoria y, de manera especial, el contenido mínimo que debe tener cada convocatoria. Con respecto a las reuniones se sugiere establecer reglas concernientes al quórum necesario para la toma de decisiones; disposiciones sobre la asistencia de los participantes, el orden del día y los lineamientos generales de lo que debe incluir la agenda; los roles específicos durante el desarrollo de las reuniones; los procedimientos para dar por terminada la reunión y los concernientes a la elaboración y validación del contenido de las actas. Además, en aras del buen funcionamiento de este órgano de gobierno, se sugiere especificar claramente las funciones y responsabilidades para el presidente y el secretario [del consejo superior o directivo]. El reglamento igualmente representa un mecanismo que permite establecer la calidad, la responsabilidad, las inhabilidades y las incompatibilidades de los [consejeros], en conjunto con los criterios de selección, los esquemas de remuneración, los principios de 
actuación y las sanciones en caso de incumplimiento de lo estipulado por el documento en relación con los deberes y responsabilidades de los [consejeros] (Guzmán y Trujillo, 2016, p. 80).

Los reglamentos de los órganos directivos igualmente permiten establecer normas sobre su composición (determinando claramente el número de externos), los comités de apoyo y su conformación y funciones, los procedimientos para evaluación de su desempeño y para inducción de nuevos miembros, y la responsabilidad del consejo en la promulgación de las tres políticas discutidas en el aparte anterior.

El segundo aspecto por incluir como recomendación para el buen funcionamiento de los órganos de gobierno en las IEs está relacionado con la necesidad de adelantar procesos de evaluación periódicos. Kiel y Nicholson (2005) afirman que la evaluación del actuar colectivo de los órganos de gobierno y de cada uno de sus miembros permite reconocer y corregir problemas de gobierno corporativo. Además, de acuerdo con estos autores, estas evaluaciones permiten establecer una cultura orientada al desempeño en la organización, permiten diferenciar claramente las funciones del ejecutivo principal y su equipo de las funciones de los órganos de gobierno, ayudan a construir mejores relaciones y a desarrollar una mejor comunicación entre los actores principales en el sistema de gobierno de la organización, y mejoran los procesos de rendición de cuentas y toma de decisiones a nivel organizacional.

Conforme con la Circular Externa 28 de 2014, hace parte de las funciones de estos órganos de gobierno organizar el proceso de evaluación anual, tanto como órgano colegiado de administración como de sus miembros individualmente considerados, de acuerdo con metodologías comúnmente aceptadas de autoevaluación o evaluación, que pueden considerar la participación de asesores externos. Este tipo de evaluaciones permite a los órganos de gobierno mejorar su eficacia, delimitar las funciones, hacer explícitas las expectativas de los consejeros, mejorar las relaciones entre ellos y con la alta dirección, evaluar la combinación de capacidades y cualidades, identificar oportunidades de capacitación, áreas de mejora y problemas que atentan contra su buen funcionamiento (Trujillo et al., 2015).

\subsection{Sobre las recomendaciones asociadas a la máxima autoridad personal de gobierno}

El Acuerdo bajo análisis resalta la importancia de contar con procesos técnicos para la selección de candidatos que ejerzan el cargo de rector de la institución. Sin embargo, es necesario mayor claridad y recomendaciones más puntuales 
al respecto. Regularmente las funciones de nominación y remuneración de altos ejecutivos y miembros de los órganos de gobierno están a cargo de un comité de gobierno designado para tal fin. Los consejos superiores o directivos pueden contar con el apoyo de comités para el ejercicio de sus funciones, los cuales generalmente adelantan funciones de auditoría, gestión de riesgos, nombramientos y retribuciones, y buenas prácticas de gobierno. Según Guzmán y Trujillo (2016), estos órganos de gobierno deben analizar las bondades de "constituir en su seno comités especializados que actúen como órganos de estudio y apoyo para materias específicas, a veces de gran complejidad técnica, y que tengan la capacidad de presentar propuestas y, eventualmente, ejercer por delegación ciertas funciones" (p. 88). Estos comités pueden ser temporales o permanentes, y representan una extensión del consejo; es decir, no son órganos independientes y consecuentemente sus funciones serán aquellas que decida el propio consejo.

Sun y Cahan (2009) y Sun, Cahan y Emanuel (2009) demuestran que los comités de nombramientos y remuneración, cuyos miembros cuentan con experiencia e independencia respecto a la alta dirección, llevan a compensaciones adecuadas para el equipo directivo, con un impacto positivo en el desempeño financiero de las organizaciones. De acuerdo con la Circular Externa 28 de 2014, el comité de nombramientos y remuneración apoya a los consejos en estas funciones, por lo que algunos de sus miembros deben contar con conocimientos en estrategia, recursos humanos (reclutamiento y selección, contratación, capacitación, administración o gestión del personal), política salarial y materias afines. Así mismo, dentro de sus funciones están las de evaluar de manera periódica las competencias, conocimientos y experiencia de los miembros de los órganos de gobierno y el equipo de alta dirección; evaluar los candidatos que pueden ejercer como la máxima autoridad personal de gobierno y proponer su nombramiento y remoción; plantear los criterios bajo los cuales se contrata y remunera a ejecutivos clave; formular la política de remuneración de los miembros del equipo directivo y de los órganos de gobierno si es el caso; y sugerir la política de recursos humanos de la IES, entre otras.

Aquí es necesario revaluar si es recomendable sugerir a las IEs constitución de algunos comités de apoyo a los consejos en tareas tan críticas como las funciones de auditoría y control interno, administración de riesgos, seguimiento a las buenas prácticas y nombramientos y remuneración. Además de las bondades resaltadas por Sun y Cahan (2009) y Sun, Cahan y Emanuel (2009) respecto al comité de compensación, otros estudios demuestran los beneficios asociados a los comités de auditoría. Por ejemplo, Xie et al. (2003) demuestran que en las organizaciones en donde se cuenta con comités de 
auditoría con una proporción de miembros expertos en finanzas la manipulación de los estados financieros es menos probable. Huang et al. (2009) expone que las organizaciones con órganos de gobierno más independientes y activos deciden conformar comités de gobierno corporativo de manera voluntaria y con ello logran mitigar el comportamiento oportunista de los ejecutivos en el interior de la organización. En general, las investigaciones en el sector empresarial han demostrado que las juntas directivas que cuentan con comités de apoyo generan un impacto positivo en el desempeño financiero de las empresas (Upadhyay et al., 2014).

\subsection{Sobre el reconocimiento de la pluralidad en los órganos de gobierno de las IES privadas}

Recientemente Bradford et al. (2018a) analizaron los órganos de gobierno en las IES privadas del país. Los autores encuentran que el $75 \%$ de las IES privadas colombianas cuentan con una especie de asamblea de representantes, un órgano de gobierno que toma nombres como asamblea general, asamblea de miembros, asamblea general de miembros, consejo de fundadores, asamblea de fundadores, sala general, sala general de miembros o cuerpo de electores. Este órgano cuenta en promedio con 16 miembros, los cuales pueden clasificarse como fundadores, adherentes o benefactores, honorarios, miembros de número, miembros elegidos por otro órgano de gobierno o escogidos entre la comunidad de la IEs. Sin embargo, en este órgano el poder de los fundadores es evidente. Se encontró mención a miembros fundadores en el $92 \%$ de las ocasiones, y ellos son los que tienen el poder de elegir directamente, o por votación mayoritaria de la asamblea, a los benefactores (mencionados en el $58 \%$ de las IES), honorarios (mencionados en el $31 \%$ de las IES), miembros de número (mencionados en el $12 \%$ de las IEs) o miembros escogidos entre aquellos que conforman la comunidad de la IEs (mencionados en el $22 \%$ de las IEs). Solo los miembros elegidos por otros órganos de gobierno no son designados por los fundadores, pero esto solo ocurre en el $4 \%$ de las IEs.

De acuerdo con Bradford et al. (2018a), estas asambleas cuentan con el poder de designar una proporción de los consejos superiores o directivos en el $94 \%$ de los casos, y en el 33\% deciden sobre la mayoría de los puestos de consejeros consagrados en los estatutos. Dentro de sus funciones están, además, las de vigilar el cumplimiento de los objetivos fundacionales ( $94 \%$ de los casos), el $83 \%$ son responsables de aprobar reformas a los estatutos, el $81 \%$ de designar el revisor fiscal y el $86 \%$ de aprobar los estados financieros anuales. Los autores señalan un ambiente de control débil, ya que solo el $10 \%$ contempla entre sus funciones el definir el sistema de control interno, 
no hay evidencia de la existencia de comités de auditoría y solo siete de las IES analizadas cuentan con un comité de nombramientos y remuneración. Pero más revelador aún es el hallazgo de Bradford et al. (2018a) respecto al poder que ostentan estos órganos de gobierno. Por ejemplo, el 61\% de estas asambleas tienen la potestad de nombrar y remover al rector, una función inherente al consejo superior o directivo, que debe asesorar y supervisar el actuar del equipo directivo. Algunas asambleas incluso tienen injerencia en aspectos académicos (20\% de los casos) y cuentan con un número de cuatro o más reuniones ordinarias al año (13\%), lo que puede indicar una interferencia en las funciones que competen al consejo y al equipo directivo. Asambleas excesivamente poderosas pueden llevar a consejos inoperantes, poco activos, decorativos, con consejeros pasivos e indiferentes con bajo nivel de compromiso (Kretek et al., 2013).

Dado la existencia de órganos de gobierno con mayor poder al de los consejos superiores o directivos, y la coexistencia de dos órganos de gobierno en las IES privadas colombianas, es imperante que una política pública para el mejoramiento de gobierno en las IEs del país reconozca esta pluralidad de órganos de gobierno y emita recomendaciones para evitar una concentración de poder en uno u otro órgano. Un buen gobierno debe garantizar un equilibrio de poderes para evitar que los miembros de estos órganos tomen ventaja del exceso de poder a fin de poner la IES al servicio de sus intereses particulares y generar situaciones de extracción de rentas, que afecten la utilización eficiente de recursos y desconozcan el bienestar común, privilegiando un interés particular. Siguiendo a Bradford et al. (2018b), como parte de las recomendaciones para un buen gobierno, es posible incluir protocolos de relacionamiento de los fundadores con las IES, en donde se hagan explícitos compromisos y reglas de interacción que permitan potenciar los beneficios del involucramiento por parte de los fundadores y mitigar posibles riesgos que puedan llevar a situaciones no deseables en las que los fundadores obtienen beneficios económicos en detrimento de la prestación del servicio de educación superior.

\subsection{Sobre la ausencia de recomendaciones asociadas a la arquitectura de control}

La arquitectura de control es un componente fundamental en el sistema de gobierno corporativo a nivel organizacional y las recomendaciones al respecto necesarias, si lo que se quiere es alentar un buen gobierno. La arquitectura o ambiente de control pretende incentivar una cultura de prevención y control 
dentro de las organizaciones, por lo que contempla los procesos de control interno, la auditoría, la administración del riesgo y la función de cumplimiento. Hay et al. (2008) afirman que el control interno y la auditoría externa, antes que sustitutos, resultan herramientas complementarias y necesarias para garantizar un ambiente de control adecuado. Krishnan y Visvanathan (2007) encuentran que cambios frecuentes en los auditores y comités de auditoría con menor proporción de expertos financieros experimentan mayores fallas en los sistemas de control interno. Además, estas fallas están relacionadas con correcciones a los estados financieros de las organizaciones.

Conforme con Trujillo y Guzmán (2017), los códigos de buen gobierno pretenden incrementar la calidad de los sistemas de gobierno y pueden desarrollarse a nivel organizacional, gremial, sectorial o a nivel país. Los primeros códigos a nivel país surgieron a partir de fallas en los sistemas de gobierno corporativo que llevaron a escándalos en los mercados de capitales. De acuerdo con Cuomo et al. (2016), el primer código nacional es el Código Cadbury, emitido en el Reino Unido en 1992. Aun cuando otros autores reconocen antecedentes previos, existe un acuerdo general en la literatura respecto a la relevancia de este, porque ha influenciado el contenido de los más de 90 códigos país en el mundo (Trujillo y Guzmán, 2017). El código resalta especialmente la importancia de los auditores, que proveen verificaciones externas y objetivas, además de la relevancia de los comités de auditoría y de los sistemas de control interno para supervisar la gestión de los recursos financieros.

La Circular Externa 28 de 2014 resalta la necesidad de establecer un ambiente de control adecuado que señale el tono y la importancia que la organización otorga al tema. Los consejos superiores o directivos son los responsables de un ambiente de control sólido, que incluye la gestión de riesgos, el sistema de control interno y los procesos de seguimiento a través de la función de auditoría. La gestión de riesgos implica la identificación de estos, su evaluación, medición de exposición, seguimiento y reporte. El sistema de control interno se encarga de la gestión de los riesgos identificados y la prevención de los problemas que pueden derivarse de ellos. Finalmente, el seguimiento a la arquitectura de control está a cargo de la función de auditoría, que internamente es ejercida por un responsable cuyo nombramiento y remoción depende directamente de los consejos, y externamente es ejercida por el revisor fiscal. Se recomienda igualmente que las organizaciones cuenten con una política para la designación del revisor fiscal y con el comité de auditoría que apoya constantemente los consejos superiores o directivos. 


\subsection{Sobre el seguimiento a la implementación de buenas prácticas en las IES colombianas}

Todos los códigos desarrollados en el ámbito global a nivel país ofrecen un conjunto de recomendaciones de implementación voluntaria, pero en la mayoría de estos se exige el reporte periódico de la implementación por parte de las organizaciones sujetas a él, y la explicación en el caso en que se decida la no adopción de una recomendación en particular (MacNeil y Li, 2006). Lo anterior genera diferentes beneficios. Primero, permite evidenciar cuáles recomendaciones se adoptan rápidamente y cuáles representan desafíos para las organizaciones. Segundo, genera disciplina por parte de las organizaciones sujetas al código. Tercero, produce un entendimiento para el emisor del código respecto a las razones de no implementar algunas recomendaciones, y si estas se sustituyen por prácticas alternativas eficientes y que atienden el principio de buen gobierno que se quiere implementar (Trujillo y Guzmán, 2017).

Consideramos que el MEN debe solicitar a las IEs un reporte anual sobre la implementación de las recomendaciones incluidas en la política pública para el mejoramiento del gobierno de las IEs. De esta manera se logrará que el Acuerdo se convierta en un instrumento efectivo de reflexión sobre las prácticas de gobierno en estas instituciones. Además, la adopción voluntaria respeta la autonomía universitaria, pero la explicación de la no adopción de algunas de las recomendaciones permite al MEN en particular, y a la sociedad en general, desarrollar control sobre las IEs como prestadoras de un servicio público de especial interés para el desarrollo económico y social del país.

\section{Comentario de cierre}

En este artículo hacemos un análisis a la evolución de la discusión sobre la importancia de las buenas prácticas de gobierno para las IEs colombianas, tanto oficiales como privadas. También, resaltamos las fortalezas de la política pública para el mejoramiento del gobierno en las IEs recientemente promulgada por el CESU. Finalmente, hacemos sugerencias puntuales de oportunidades para fortalecer la política en mención. Específicamente, proponemos recomendar la elaboración de las políticas para el manejo de los conflictos de intereses, de transacciones con partes relacionadas y de relevación de información, todas ellas responsabilidad de los consejos superiores y directivos. Además, resaltamos la relevancia de los reglamentos de los consejos, como una herramienta que potencia su correcto funcionamiento, así como las bondades de implementar prácticas de evaluación del actuar colectivo 
e individual de estos órganos de gobierno, para identificar áreas de mejora. De igual forma, resaltamos el aporte de los comités que se pueden constituir en el seno de los consejos, en especial, del comité de nombramientos y remuneración para la selección, compensación y evaluación del desempeño del rector y su equipo, así como del comité de auditoría, como parte de la arquitectura de control de las IEs. Por otra parte, señalamos la importancia de tener en cuenta la pluralidad en los órganos de gobierno de las IEs privadas y la necesidad de ofrecer recomendaciones que lleven a un equilibrio de poderes entre los distintos órganos de gobierno. Y, para terminar, sugerimos ofrecer recomendaciones dirigidas a contar con un ambiente y arquitectura de control adecuados para las IEs, al igual que implementar mecanismos de reporte por parte de las instituciones que informen al MEN sobre el avance en la implementación de las recomendaciones y las razones o sustento de las IEs en el caso en que decidan no adoptar una recomendación en especial. Consideramos que estos aspectos continuarán consolidando el camino del país hacia IES gobernadas de la manera adecuada, y que, gracias a lo anterior, estén en capacidad de prestar un servicio público enmarcado en altos estándares de calidad y eficiencia.

Es posible que el lector, aun después de leer este artículo, se esté preguntando por qué se habla de gobierno corporativo en las IEs colombianas, las cuales, oficiales o privadas, por ley son instituciones sin ánimo de lucro. Es decir, primero, no son empresas o corporaciones con socios o accionistas, y no persiguen la generación de utilidades para distribución entre aquellos que apoyaron su constitución. Por lo anterior, es necesario aclarar que seguimos los planteamientos de Bradford et al. (2018a) y de Restrepo et al. (2012). Para estos autores, el uso del marco teórico del gobierno corporativo en el contexto del gobierno de las IEs implica el reconocimiento de la existencia de conflictos de interés y tensiones de agencia en el interior de estas instituciones y la necesidad de mitigarlos para garantizar la efectividad de estas instituciones y el uso adecuado de sus recursos. Invitamos especialmente al lector a leer la segunda sección del artículo de Bradford et al. (2018a), "Gobierno de la educación superior y gobierno corporativo".

Entendemos que la responsabilidad primaria de las IEs está en la generación de bienestar social a través de la prestación del servicio público de la educación superior, bajo estándares de calidad y eficiencia. Por lo tanto, el gobierno de las IEs debe ser efectivo y legítimo, generando bienestar para la sociedad y teniendo en cuenta las necesidades e intereses de los diferentes grupos de interés (Leslie, 1975). Desde esta perspectiva teórica, la adecuada gestión de los conflictos de interés se hace pertinente e imprescindible, para evitar pérdidas económicas, una inadecuada administración de los recursos 
$\mathrm{y}$ un deterioro de las condiciones bajo las cuales las IES prestan el servicio de educación superior. Esto ha sido entendido por aquellos que realizan sus estudios sobre las prácticas de gobierno corporativo en las IES, como MéndezBeltrán (2015), De Silva y Armstrong (2015), Zaman (2015), Das y Pattanayak (2016) y Flórez-Parra et al. $(2017,2018)$.

\section{Referencias}

Akerlof, G. (1970). The market for lemons: quality uncertainty and the market mechanism. Quarterly Journal of Economics, 84(3), 488-500.

Andrews, K. (1971). The concept of corporate strategy. Homewood, IL: Dow Jones-Irwin. (2a ed., 1980; $3^{\text {a }}$ ed., 1987).

Bertrand, M., \& Schoar, A. (2006). The role of family in family firms. Journal of Economic Perspectives, 20(2), 73-96.

Bradford, H., Guzmán, A., Restrepo, J. M., \& Trujillo, M. A. (2018a). Who controls the board in non-profit organizations? The case of private higher education institutions in Colombia. Higher Education, 75(5), 909-924.

Bradford, H., Guzmán, A., Restrepo, J. M., \& Trujillo, M. A. (2018b). Family involvement in higher education institutions: a matter of governance. Documento de investigación. Bogotá: Colegio de Estudios Superiores de Administración (CESA).

Católico, D. F. (2012). Revelación y divulgación de la información financiera y no financiera de las universidades públicas en Colombia. Revista Facultad de Ciencias Económicas: Investigación y Reflexión, 20(1), 57-76.

Coffee, J. (1999). The future as history: the prospects for global convergence in corporate governance and its implications. Northwestern University Law Review, 93(3), 641-707.

Colombia, Congreso de la República. Ley 1740 de 2014, por la cual se desarrolla parcialmente el artículo 67 y los numerales 21, 22 y 26 del artículo 189 de la Constitución Política, se regula la inspección y vigilancia de la educación superior, se modifica parcialmente la Ley 30 de 1992 y se dictan otras disposiciones. Diario Oficial, 23 de diciembre de 2014.

Colombia, Congreso de la República. Ley 30 de 1992, por la cual se organiza el servicio público de la educación superior. Diario Oficial, 28 de diciembre de 1992.

Colombia, Consejo Nacional de Educación Superior (CEsu). Acuerdo 2 de 2017, por medio del cual se establece la política pública para el mejoramiento del gobierno de las instituciones de educación superior (8 noviembre 2017). 
Colombia, Superintendencia Financiera. Circular Externa 28 de 2014. Presentación del nuevo código país y adopción del reporte de implementación de mejores prácticas corporativas (30 septiembre 2014).

Corbally, J. E., \& Sergiovanni, T. J. (Eds.). (1984). Leadership and organizational culture: new perspectives on administrative theory and practice. University of Illinois Press.

Cuomo, F., Mallin, C., \& Zattoni, A. (2016). Corporate governance codes: a review and research agenda. Corporate Governance: An International Review, 24(3), 222-241.

Das, N., \& Pattanayak, J. K. (2016). Corporate governance mechanism for academic institutions imparting higher education in India. International Journal of Management in Education, 10(2), 204-217.

De Silva Lokuwaduge, C., \& Armstrong, A. (2015). The impact of governance on the performance of the higher education sector in Australia. Educational Management Administration E Leadership, 43(5), 811-827.

Denis, D. K. (2001). Twenty-five years of corporate governance research... and counting. Review of Financial Economics, 10(3), 191-212.

Fama, E., \& Jensen, M. (1983). Agency problems and residual claims. The Journal of Law and Economics, 26(2), 327-349.

Flórez-Parra, J. M. (2015). El gobierno corporativo en el sector público: un estudio aplicado a las universidades colombianas (Tesis doctoral, Universidad de Granada, España).

Flórez-Parra, J. M., López-Pérez, M. V., \& López-Hernández, A. M. (2017). Transparency and its determinants at Colombian universities. Higher Education Research E Development, 36(4), 674-687.

Flórez-Parra, J. M., López-Pérez, M. V., \& López-Hernández, A. M. (2018). Corporate governance in Colombian universities. International Review of Administrative Sciences, en proceso de publicación.

Freeman, R. (1984). Strategic management: a stakeholder approach. Boston: Pitman. Gordon, E. A., Henry, E., \& Palia, D. (2004). Related party transactions and corporate governance. En M. Hirschey, J. Kose \& M. Anil K. (Eds.), Corporate governance (advances in financial economics, volume 9) (pp. 1-27). Emerald Group Publishing Limited.

Guzmán, A., \& Trujillo, M. A. (2016). Lineamientos de gobierno corporativo en revelación y transparencia. Bogotá: Colegio de Estudios Superiores de Administración (CESA).

Hay, D., Knechel, W. R., \& Ling, H. (2008). Evidence on the impact of internal control and corporate governance on audit fees. International Journal of Auditing, 12(1), 9-24. 
Hendry, K., \& Kiel, G. (2004). The role of the board in firm strategy: integrating agency and organisational control perspectives. Corporate Governance: An International Review, 12(4), 500-520.

Huang, H., Lobo, G. J., \& Zhou, J. (2009). Determinants and accounting consequences of forming a governance committee: evidence from the United States. Corporate Governance: An International Review, 17(6), 710-727.

Huse, M. (2000). Boards of directors in smes: a review and research agenda. Entrepreneurship \& Regional Development, 12(4), 271-290.

Jensen, M., \& Meckling, W. (1976). Theory of the firm: managerial behavior, agency costs and ownership structure. Journal of Financial Economics, 3(4), 305-360.

Kiel, G. C., \& Nicholson, G. J. (2005). Evaluating boards and directors. Corporate Governance: An International Review, 13(5), 613-631.

Kretek, P. M., Dragšić, Ž., \& Kehm, B. M. (2013). Transformation of university governance: on the role of university board members. Higher Education, 65(1), 39-58.

Krishnan, G. V., \& Visvanathan, G. (2007). Reporting internal control deficiencies in the post-Sarbanes-Oxley era: the role of auditors and corporate governance. International Journal of Auditing, 11(2), 73-90.

Leslie, D.W. (1975). Legitimizing university governance: theory and practice. Higher Education, 4(2), 233-246.

MacNeil, I., \& Li, X. (2006). 'Comply or explain': market discipline and noncompliance with the combined code. Corporate Governance: An International Review, 14(5), 486-496.

MEN \& PUJ. (2014). Recomendaciones para una política pública sobre buen gobierno universitario en Colombia. Bogotá: Ministerio de Educación Nacional de Colombia-Escuela Javeriana de Gobierno y Ética Pública de la Pontificia Universidad Javeriana.

MEN. (2013). Guía para la creación e implementación de códigos de buen gobierno en las instituciones de educación superior. Bogotá: Ministerio de Educación Nacional de Colombia.

Méndez-Beltrán, J. A. (2015). Divulgación on-line de los códigos de buen gobierno en las IES y su determinación para el posicionamiento en el ranking QS (Tesis de maestría, Universidad del Rosario, Bogotá, Colombia).

Myers, S. (1977). Determinants of corporate borrowing. Journal of Financial Economics, 5(2), 147-175.

Nekhili, M., \& Cherif, M. (2011). Related parties transactions and firm's market value: the French case. Review of Accounting and Finance, 10(3), 291-315. OCDE. (2008). Tertiary education for the knowledge society. Volume 1. París: ocDE. 
Prada-Rangel, J. A. (2014). Modelo de prospección financiera operacional, como una herramienta estratégica y de gestión de los gobiernos corporativos de las IES privadas en Colombia (Tesis de maestría, Colegio de Estudios Superiores de Administración, CEsA, Bogotá, Colombia).

Restrepo, J., Trujillo, M., \& Guzmán, A. (2012). Gobierno corporativo en las instituciones de educación superior en Colombia. Bogotá: Colegio de Estudios Superiores de Administración (CESA)-Ministerio de Educación Nacional de Colombia.

Shleifer, A., \& Vishny, R. (1986). Large shareholders and corporate control. Journal of Political Economy, 94(3, Part 1), 461-488.

Siciliano, J. (2005). Board involvement in strategy and organizational performance. Journal of General Management, 30(4), 1-10.

Spence, M. (1973). Job market signaling. The Quarterly Journal of Economics, 87(3), 355-374.

Spence, M. (1974). Market signaling: informational transfer in hiring and related screening processes. Cambridge, ma: Harvard University Press.

ssoc. (2009). Guía colombiana de gobierno corporativo para sociedades cerradas y de familia. Bogotá: Superintendencia de Sociedades-Confecámaras-Cámara de Comercio de Bogotá.

Sun, J., \& Cahan, S. (2009). The effect of compensation committee quality on the association between CEO cash compensation and accounting performance. Corporate Governance: An International Review, 17(2), 193-207.

Sun, J., Cahan, S. F., \& Emanuel, D. (2009). Compensation committee governance quality, chief executive officer stock option grants, and future firm performance. Journal of Banking E Finance, 33(8), 1507-1519.

Tirole, J. (2001). Corporate governance. Econometrica, 69(1), 1-35.

Trujillo, M. A., \& Guzmán, A. (2015). Revelación de información y valor de las empresas en América Latina. Bogotá: Colegio de Estudios Superiores de Administración (CESA).

Trujillo, M. A., \& Guzmán, A. (2017). Emisores de valores E gobierno corporativo: un análisis a las encuestas de código país 2007-2014. Bogotá: Colegio de Estudios Superiores de Administración (CESA).

Trujillo, M. A., Guzmán, A. \& Prada, F. (2015). Juntas directivas en el desarrollo del gobierno corporativo. Bogotá: Colegio de Estudios Superiores de Administración (CESA).

Upadhyay, A., Bhargava, R., \& Faircloth, S. (2014). Board structure and role of monitoring committees. Journal of Business Research, 67(7), 1486-1492.

Villalonga, B., Amit, R., Trujillo, M. A., \& Guzmán, A. (2015). Governance of family firms. Annual Review of Financial Economics, 7, 635-654. 
Xie, B., Davidson III, W. N., \& DaDalt, P. J. (2003). Earnings management and corporate governance: the role of the board and the audit committee. Journal of Corporate Finance, 9(3), 295-316.

Zaman, K. (2015). Quality guidelines for good governance in higher education across the globe. Pacific Science Review B: Humanities and Social Sciences, 1(1), 1-7. 